
Not for reproduction, distribution or commercial use.

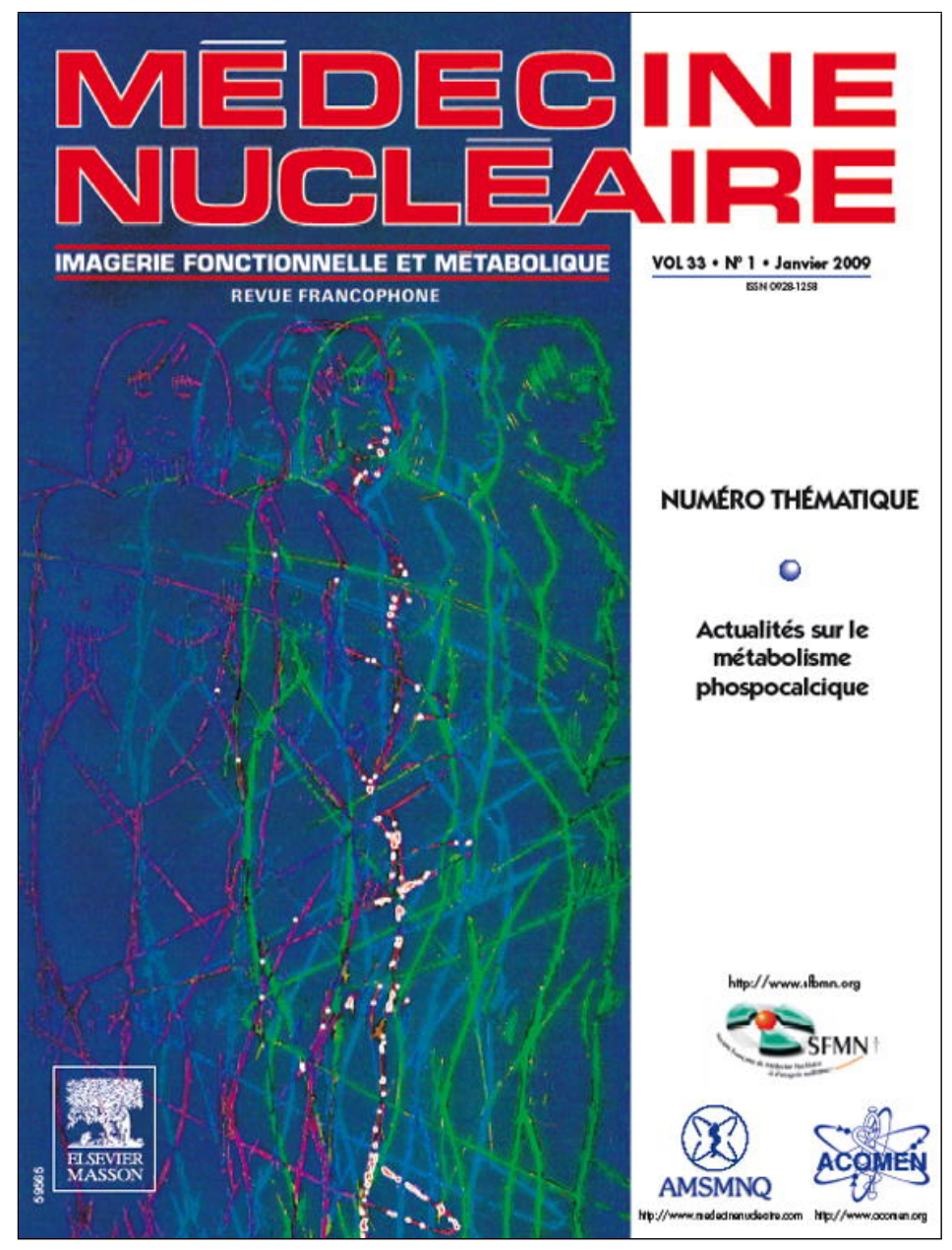

This article appeared in a journal published by Elsevier. The attached copy is furnished to the author for internal non-commercial research and education use, including for instruction at the authors institution and sharing with colleagues.

Other uses, including reproduction and distribution, or selling or licensing copies, or posting to personal, institutional or third party websites are prohibited.

In most cases authors are permitted to post their version of the article (e.g. in Word or Tex form) to their personal website or institutional repository. Authors requiring further information regarding Elsevier's archiving and manuscript policies are encouraged to visit:

http://www.elsevier.com/copyright 
Mise au point

\title{
La vitamine D : effets « classiques », « non classiques » et évaluation du statut du patient
}

\section{An update on the classical and non classical effects on vitamin D; evaluation of the patient's status}

\author{
E. Cavalier ${ }^{\mathrm{a}, *}$, J.-C. Souberbielle ${ }^{\mathrm{b}}$ \\ ${ }^{a}$ Service de chimie médicale, université de Liège, CHU de Liège, domaine du Sart-Tilman, 4000 Liège, Belgique \\ ${ }^{\mathrm{b}}$ Service d'explorations fonctionnelles, hôpital Necker-Enfants-malades, AP-HP, Paris, France
}

Reçu le 29 septembre 2008 ; accepté le 9 octobre 2008

Disponible sur Internet le 6 janvier 2009

\section{Résumé}

Les connaissances sur la vitamine $\mathrm{D}$ ont beaucoup progressé ces dernières années. La vitamine $\mathrm{D}$ ne peut plus être considérée comme uniquement nécessaire à la prévention du rachitisme/ostéomalacie. Son rôle dans la prévention des fractures ostéoporotiques périphériques du sujet âgé est maintenant bien démontré (en association avec du calcium) et de très nombreuses données épidémiologiques et expérimentales sont en faveur d'un rôle dans la prévention de nombreuses affections (certains cancers et maladies auto-immunes, évènements cardiovasculaires et hypertension, sarcopénie du sujet âgé...). Déjà, quelques études d'intervention confirment certains de ces effets. L'évaluation du statut vitaminique $\mathrm{D}$ peut être aisément réalisée par le dosage de la $25-\mathrm{OH}$ vitamine $\mathrm{D}$ sérique. Toutefois, la plupart des revues récentes sur le sujet suggèrent que les valeurs de référence de la $25-\mathrm{OH}$ vitamine $\mathrm{D}$ obtenues dans des populations apparemment en bonne santé sont beaucoup trop basses et que la concentration de $25-\mathrm{OH}$ vitamine D au-dessous de laquelle il existe une insuffisance en vitamine D se situe entre 50 et $100 \mathrm{nmol} / \mathrm{L}$ ( $20 \mathrm{et} 40 \mathrm{ng} / \mathrm{mL}$ ) avec une franche tendance à cibler des valeurs supérieures à $75 \mathrm{nmol} / \mathrm{L}(30 \mathrm{ng} / \mathrm{mL})$. Les supplémentations habituellement recommandées sont insuffisantes pour atteindre ces concentrations.

(C) 2008 Elsevier Masson SAS. Tous droits réservés.

\begin{abstract}
Knowledge about vitamin D has greatly improved during the last few years. Vitamin D cannot any more be considered as exclusively necessary to prevent ricket/osteomalacia. Its role in the prevention of some osteoporotic fractures in the elderly (in association with calcium nutrition) is now well-demonstrated and many epidemiologic and laboratory data argue for a role in the prevention of several diseases or anomalies (cancer, autoimmune diseases, cardiovascular events, sarcopenia...). A few intervention studies confirming some of these effects also exist. Vitamin D status can easily be assessed by measuring serum $25-\mathrm{OH}$ vitamin D level. However, many experts have claimed that the population-based reference values for $25 \mathrm{OHD}$ are too low and that the cut-off value below which vitamin D insufficiency can be present is somewhere between $20 \mathrm{and} 40 \mathrm{ng} / \mathrm{mL}$ with a clear tendency to target values above $30 \mathrm{ng} / \mathrm{mL}$ ( $75 \mathrm{nmol} / \mathrm{L})$. The main consequences are that vitamin D insufficiency is highly frequent whereas the currently recommended supplementation doses are not sufficient.
\end{abstract}

(C) 2008 Elsevier Masson SAS. All rights reserved.

Mots clés : Vitamine D ; 25 hydroxy vitamine D ; Hormone parathyroïdienne ; Ostéoporose ; Cancer

Keywords: Vitamin D; 25 hydroxy vitamin D; Parathyroid hormone; Osteoporosis; Cancer

\footnotetext{
* Auteur correspondant.

Adressee-mail : etienne.cavalier@chu.ulg.ac.be (E. Cavalier).
} 


\section{Introduction}

La vitamine $\mathrm{D}$ est très importante pour la croissance et la santé osseuse. Le terme vitamine est inapproprié pour la vitamine $\mathrm{D}$ qui doit être plutôt considérée comme une prohormone. En effet, la peau peut la synthétiser à partir du 7-dehydrocholesterol sous l'effet de certains rayonnements UVB (dont la longueur d'onde est comprise entre 290 et $315 \mathrm{~nm}$ ) [1]. En situation d'exposition intense à un ensoleillement important, l'excès de prévitamine D3 formé est transformé en composé inactif et il n'y a pas d'intoxication à la vitamine $\mathrm{D}$ suite à une exposition trop forte au soleil. La vitamine $\mathrm{D}$ existe sous deux formes, la vitamine D3 (cholecalciférol) qui est la molécule synthétisée par la peau sous l'influence des UVB ou retrouvée dans les rares sources alimentaires animales (poissons gras en particulier) et la vitamine D2 (ergocalciférol) qui est la vitamine D des plantes. Parmi les spécialités médicamenteuses, certaines sont de la vitamine D2 et d'autres sont de la vitamine D3. Qu'elle soit synthétisée par la peau ou apportée par l'alimentation ou la supplémentation, la vitamine D (D2 ou D3) est transportée dans le sang par une protéine porteuse, la « vitamin D binding protein » (DBP), jusqu'au foie où elle est hydroxylée sur le carbone 25 pour former la 25 hydroxy-vitamine D (25OHD). Cette hydroxylation n'est pas régulée, c'est-à-dire que plus la quantité de vitamine D synthétisée ou ingérée est importante, plus la quantité de 25OHD formée est grande. Cependant, dans le cadre de traitements par la vitamine $\mathrm{D}$, plusieurs études ont trouvé une relation négative entre l'augmentation de la concentration de $25 \mathrm{OHD}$ et sa concentration de départ [2]. La 25OHD circule dans le sang avec une demi-vie de l'ordre de 3-4 semaines. Elle entre dans les cellules du tubule proximal rénal, soit sous sa forme libre (non liée à la DBP), soit associée à la DBP en se liant à une protéine de surface, la mégaline [3]. Grâce à une enzyme, la 1-alpha hydroxylase (CYP27B1), la $25 \mathrm{OHD}$ y est hydroxylée sur le carbone-1 pour former la 1,25 dihydroxy vitamine D $[1,25(\mathrm{OH}) 2 \mathrm{D}]$ ou calcitriol. Cette hydroxylation rénale est étroitement régulée et est stimulée principalement par la PTH, par une hypophosphatémie ou de faibles apports alimentaires en calcium, et est inhibée par le FGF-23 et une hyperphosphatémie [1]. La 1,25(OH)2D est le métabolite actif de la vitamine $\mathrm{D}$ et sa demi-vie dans le sérum est de quatre heures environ. Il existe par ailleurs une voie d'inactivation de la vitamine D via une enzyme, la 24 hydroxylase (CYP24) qui induit la production de composés inactifs [24,25 $(\mathrm{OH}) 2$ vitamine $\mathrm{D}, 1,24,25(\mathrm{OH}) 3$ vitamine $\mathrm{D}]$ transformés ensuite en acide calcitroïque inactif.

\section{Effets de la vitamine D}

La 1,25(OH)2D agit via un récepteur cytosolique, le VDR, présent dans de nombreux tissus [1]. La 1,25(OH)2D peut exercer des effets endocrines (la $1,25(\mathrm{OH}) 2 \mathrm{D}$ produite par le rein est transportée dans le sang jusqu'à ses tissus cibles), ou des effets qu'on peut qualifier d'autocrines (de nombreux tissus expriment la 1-alpha-hydroxylase ainsi que le VDR. La 25OHD pénètre dans ces tissus et y est hydroxylée en $1,25(\mathrm{OH}) 2 \mathrm{D}$ qui ne « ressort » pas de la cellule et y agit localement, l'excès étant métabolisé en composé inactif). Dans la cellule, la 1,25(OH)2D se lie au VDR. Le complexe VDR-1,25(OH)2D est « dirigé » vers le noyau de la cellule où il s'associe au récepteur de l'acide rétinoïque (RXR). Le complexe RXR-VDR-1,25(OH)2D se lie à l'ADN en des sites appelés "éléments de réponse à la vitamine $\mathrm{D} »$ (VDRE), proches de gènes dont l'expression est ainsi soit activée soit réprimée, ce qui module la synthèse de nombreuses protéines [1].

\subsection{Effets « classiques » de la vitamine $D$}

Le rôle le mieux connu de la 1,25(OH)2D est le maintien de l'homéostasie phospho-calcique par augmentation de l'absorption intestinale du calcium et du phosphore. Dans la cellule intestinale, la 1,25(OH)2D induit la synthèse de la protéine TRPV6 (qui crée un canal calcique au niveau de la bordure en brosse apicale de l'entérocyte permettant l'entrée de calcium dans la cellule), de la calbindine 9K (qui transporte le calcium dans l'entérocyte) [4] et de la protéine NPT2b (qui est un co-transporteur sodium-phosphate favorisant l'entrée de phosphate dans l'entérocyte) [5]. Ce processus actif est prépondérant lorsque les apports calciques ou phosphorés sont faibles ou dans des conditions physiologiques (croissance, grossesse) ou pathologiques (granulomatoses, hyperparathyroïdies...) où la concentration plasmatique de 1,25(OH)2D est élevée. Il permet d'augmenter significativement la fraction de calcium et de phosphate absorbée par rapport à la quantité ingérée. Cela va favoriser un environnement minéral optimal pour le tissu osseux et permettre la minéralisation osseuse (on ne peut toutefois pas dire que la vitamine $\mathrm{D}$ « fixe » le calcium). Un déficit profond en vitamine $\mathrm{D}$ peut ainsi avoir pour conséquence des pathologies osseuses caractérisées par un défaut de minéralisation, rachitisme chez l'enfant, ostéomalacie chez l'adulte. Cela est particulièrement fréquent lorsque ce déficit est associé à une malabsorption [6]. Lorsque le déficit en vitamine D est moins profond, il n'y a pas de troubles de la minéralisation, mais la diminution de l'absorption intestinale du calcium et la tendance hypocalcémique qui s'ensuit induisent une élévation de la concentration de PTH qui stimule le remodelage osseux et qui, à long terme, contribue à l'ostéoporose du sujet âgé. Dans les ostéoblastes, la $1,25(\mathrm{OH}) 2 \mathrm{D}$ stimule la production de RANKL, cytokine stimulant la résorption par les ostéoclastes. Enfin, la 1,25(OH)2D exerce un retrocontrôle négatif de la sécrétion de PTH par les parathyroïdes, limitant ainsi l'hyperplasie des parathyroïdes en cas d'hyperparathyroïdie.

Quelques données, principalement obtenues dans des études d'observation, suggèrent une relation positive entre les apports en vitamine $\mathrm{D}$ et la densité minérale osseuse chez les adolescents et les jeunes adultes, ce qui est intéressant pour l'optimisation du pic de masse osseuse. Plusieurs études d'intervention (randomisées, en double insu contre placebo) ont évalué l'effet d'une supplémentation en vitamine D associée ou non à du calcium sur la diminution du risque de fracture périphérique. Une meta-analyse de 2005 a conclu à une réduction du risque relatif de fracture si la dose de vitamine $\mathrm{D}$ 
administrée était supérieure à 700-800 UI/J (aucun effet antifracturaire n'était noté dans les études qui ont utilisé des doses de $400 \mathrm{UI} / \mathrm{J}$ ) [7]. Une meta-analyse plus récente qui a pourtant intégré deux très grandes études négatives, a également conclu à l'efficacité de la vitamine $\mathrm{D}$ (à des doses d'au moins $800 \mathrm{UI} / \mathrm{J}$ ) pour réduire le risque de fracture chez les sujets de plus de 50 ans, en particulier lorsqu'elle était associée à du calcium (1200 mg de calcium par jour) [8]. Dans cette meta-analyse, les résultats ont montré que cet effet antifracturaire était plus prononcé chez les sujets de plus de 70 ans que chez ceux de 50 à 70 ans, ainsi que chez les sujets qui avaient une concentration basse de vitamine $\mathrm{D}$ au début de l'étude. Enfin, et même si cela peut paraître évident (encore fallait-il le démontrer!), le risque de fracture était d'autant plus diminué que l'adhérence au traitement était bonne.

\subsection{Effets « non classiques » de la vitamine $D$}

La répartition géographique traditionnelle de l'incidence du rachitisme/ostéomalacie est bien connue. En l'absence de supplémentation, ces pathologies sont plus fréquentes dans les pays recevant un faible ensoleillement. Une répartition géographique similaire a été identifiée pour un certain nombre de pathologies comme le diabète de type I, la sclérose en plaques ou les cancers de la prostate, du côlon et du sein [911]. L'hypothèse que l'insuffisance en vitamine $D$ pouvait avoir un rôle dans la genèse de ces maladies ou d'autres comme l'hypertension artérielle [12] ou l'insuffisance cardiaque [13] a donc été émise. Alors que le déficit en vitamine $\mathrm{D}$ a traditionnellement été défini en termes d'effets osseux, de nombreux tissus n'ayant rien à voir avec le métabolisme de l'os ou du calcium, possèdent le VDR et répondent à la $1,25(\mathrm{OH}) 2 \mathrm{D}$ (par exemple les cellules de la prostate ou du tissu hématopoḯtique). Toutefois, alors que les concentrations de 25OHD sériques varient fortement avec la latitude ou la saison, les concentrations de 1,25(OH)2D sont relativement stables (grâce à une hyperparathyroïdie secondaire lorsque la $25 \mathrm{OHD}$ est trop basse [14,15]) et l'effet endocrine de la vitamine D ne peut donc pas expliquer cette variabilité géographique dans l'incidence de ces maladies. En fait, comme indiqué plus haut, ces cellules expriment la 1-alpha hydroxylase, qui permet la synthèse de $1,25(\mathrm{OH}) 2 \mathrm{D}$ à partir de la 25OHD, et utilisent la $250 H D$ circulante pour fabriquer leur propre $1,25(\mathrm{OH}) 2 \mathrm{D}$ qui agit ensuite de manière autocrine (ou « intracrine »). Les déterminants de la 1-alpha hydroxylase extrarénale sont probablement différents de ceux de l'enzyme rénale et le niveau de vitamine D optimal pour ces organes est inconnu mais est probablement supérieur au niveau requis pour éviter les pathologies osseuses $[1,16]$. On peut donc supposer que certaines pathologies puissent être influencées (au moins en partie) par une insuffisance en vitamine D, même en l'absence de signes osseux d'insuffisance en vitamine $\mathrm{D}$.

\subsubsection{Vitamine $D$ et fonction musculaire}

Il est bien connu que le rachitisme/ostéomalacie s'accompagne de douleurs et faiblesse musculaires. Plusieurs études d'observation ont retrouvé une association très significative entre des concentrations sériques basses de 250HD et une sarcopénie, en particulier chez les sujets âgés [17]. Cela ne veut toutefois pas dire qu'il y a une relation de causalité et la 25OHD basse pourrait n'être qu'un marqueur d'un mauvais état général. Cependant, plusieurs études d'intervention ont montré qu'une supplémentation en vitamine D améliorait les performances musculaires des sujets âgés carencés (facilité à se lever d'une chaise sans l'aide des mains, amélioration de la vitesse de déplacement sur une courte distance) [18] et réduisait le risque relatif de chutes (ce qui explique en partie la diminution du risque de fractures périphériques) [19]. Cet effet bénéfique de la vitamine $\mathrm{D}$ pourrait être lié d'une part à un effet direct sur la taille des fibres musculaires de type-2 [20], et d'autre part à une activation de la protéine kinase $\mathrm{C}$ qui favorise l'augmentation du pool calcique intracellulaire nécessaire à la contraction musculaire [21].

\subsubsection{Vitamine $D$ et cancers}

De très nombreuses études « écologiques » ont montré une relation entre latitude et prévalence de nombreux cancers (plus on s'éloigne de l'équateur, plus certains cancers sont fréquents [10,11,22-24]). De très nombreuses études d'observation (études cas-témoin ou études prospectives de cohorte) ont montré que les sujets qui avaient les plus forts apports en vitamine $\mathrm{D}$ et/ou les plus fortes concentrations de 25OHD, avaient un risque relatif de cancers (surtout colorectaux [25-28], mais aussi prostate [29], pancréas [30], poumon [31]...) significativement plus faible (souvent environ de moitié) que les sujets qui, au contraire, avaient des faibles apports de vitamine $\mathrm{D}$ et/ou des concentrations de 25OHD basses. Alors qu'une étude chez 36000 femmes américaines qui ont reçu soit $400 \mathrm{U}$ de vitamine $\mathrm{D}$ et un gramme de calcium par jour, soit un placebo, n'a pas montré de réduction du risque de cancer du colôn [32], une étude plus récente chez plus de 1100 femmes ménopausées ayant reçu soit un placebo, soit du calcium, soit du calcium + de la vitamine $\mathrm{D}$ à la dose (plus forte que d'habitude) de $1100 \mathrm{U} / \mathrm{J}$, a montré une réduction de $60 \%$ environ du risque de cancers (différents cancers) dans le groupe calcium + vitamine D par rapport au groupe placebo [33]. Notons toutefois que, d'une part, cette étude n'était pas initialement prévue pour étudier le risque de cancer, et que, d'autre part, le nombre de cas de cancers $(20 / 288$ soit $6,9 \%$ dans le groupe placebo et $13 / 446$ soit $2,9 \%$ dans le groupe calcium + vitamine D) était faible. Ces résultats nécessitent donc d'être confirmés dans de nouvelles études qui utiliseront des doses «fortes » de vitamine D avec et sans calcium, pour des durées les plus longues possibles. En se basant sur de nombreuses études expérimentales (culture cellulaire, modèles animaux...), il apparaît que l'explication la plus probable de cet effet antitumoral est lié au fait que dans certains tissus, la $1,25(\mathrm{OH}) 2 \mathrm{D}$ régule un certain nombre de gènes qui contrôlent la prolifération cellulaire, et stimule d'autres gènes qui, eux, inhibent l'angiogénèse et induisent l'apoptose des cellules tumorales [34-38]. 


\subsubsection{Vitamine $D$ et système immunitaire}

Globalement, de nombreuses études expérimentales sont en faveur d'une inhibition de l'immunité acquise et d'une stimulation de l'immunité innée par la vitamine D. Le VDR et la 1-alpha-hydroxylase sont présents dans les lymphocytes T et $\mathrm{B}$, les macrophages et les cellules présentatrices d'antigène. Les études montrent que la $1,25(\mathrm{OH}) 2 \mathrm{D}$ réduit la prolifération lymphocytaire et la production de certaines cytokines [39] et peut atténuer ou même prévenir différentes maladies dans des modèles animaux, principalement murins d'encéphalomyélite (modèle de la sclérose en plaques) [40], de lupus [41], de polyarthrite rhumatoïde [42], de diabète de type-1 [43]. . . Le mécanisme de ces effets immunorégulateurs est en partie lié à la stimulation de la synthèse de TGF $\beta 1$ et d'IL4 [44] et nécessite la présence de calcium [45]. Ces résultats sont cohérents avec ceux de nombreuses études épidémiologiques montrant une relation entre une plus grande fréquence de certaines maladies auto-immunes, diabète de type-1 [46,47], sclérose en plaques [48], polyarthrite rhumatoïde [49] et, soit de faibles apports de vitamine $\mathrm{D}$, soit des concentrations basses de 25OHD. Concernant l'immunité innée, on sait maintenant que les macrophages ou les monocytes exposés à un agent infectieux comme le bacille de la tuberculose, surexpriment le «Toll-like receptor-2», le VDR et la 1-alpha hydroxylase. À condition que la concentration de 25OHD dans le liquide extracellulaire de ces cellules soit suffisante, elles vont former de la $1,25(\mathrm{OH}) 2 \mathrm{D}$ qui va induire la production de protéines, en particulier la cathélicidine, que l'on peut considérer comme des antibiotiques naturels, et qui vont détruire l'agent infectieux [50]. Ce mécanisme explique (au moins en partie) la relation entre la fréquence de la tuberculose et des concentrations basses de $25 \mathrm{OHD}$ retrouvée dans des études épidémiologiques [51].

\subsubsection{Vitamine D et système cardiovasculaire}

Plusieurs études d'observation rapportent une association entre risque cardiovasculaire et concentrations basses de 25OHD [11,52], mais la relation de causalité n'est pas définitivement établie. Certaines de ces études (mais pas toutes) ont confirmé cette association après ajustement statistique pour des facteurs confondants comme l'obésité, l'inactivité physique, la consommation d'alcool ou de tabac. Ce point est important car ces facteurs sont tous des facteurs de risque cardiovasculaires et certains (l'obésité et la sédentarité) sont particulièrement associés à des concentrations basses de 25OHD. Parmi les mécanismes qui pourraient expliquer un effet de la vitamine D sur le risque cardiovasculaire, on peut noter :

- un effet de la vitamine D sur la pression artérielle. En effet, la $1,25 \mathrm{OH} 2 \mathrm{D}$ contrôle l'expression du gène de la rénine [53] et les souris dont le gène du VDR est invalidé ont une hypertension artérielle avec rénine élevée. Des études d'intervention ont montré qu'une exposition à des rayonnements UVB (versus des UVA) [54] ou la prise de vitamine D (versus un placebo) [12] pouvait réduire significativement la pression artérielle chez des patients hypertendus ;
- un effet possible sur le risque de diabète de type- 2 via une amélioration de la sensibilité à l'insuline et de l'intolérance au glucose [55].

\section{3. Évaluation du statut vitaminique D}

Même si la 1,25(OH)2D est unanimement reconnue comme le métabolite actif de la vitamine $\mathrm{D}$, plusieurs études suggèrent d'importantes fonctions physiologiques pour la 25OHD. D'une part, elle est le substrat pour la formation de 1,25(OH)2D et d'autre part elle semble avoir une activité directe sur l'absorption intestinale du calcium, 200 à 1000 fois plus faible (suivant les études) que celle de la 1,25(OH)2D, mais avec des concentrations circulantes 500 à 1000 fois plus élevées [56]. De plus, les parathyroïdes sont elles-même capables de transformer localement la $25 \mathrm{OHD}$ en $1,25(\mathrm{OH}) 2 \mathrm{D}$ qui pourrait alors moduler la sécrétion de PTH [95]. La conséquence de cela est qu'un déficit modéré en vitamine D (appelé de plus en plus souvent une insuffisance en vitamine D) entraînera une diminution de l'absorption intestinale du calcium et une tendance hypocalcémique, elle-même compensée par une élévation de la PTH [14]. Cette hyperparathyroïdie secondaire va stimuler la 1-alpha hydroxylase, augmentant la concentration sérique de 1,25(OH)2D. Dans une insuffisance en vitamine $\mathrm{D}$, la 1,25(OH)2D sérique pourra être normale, élevée ou basse [1]. La mesure de la 1,25(OH)2D n'est donc pas appropriée pour évaluer le statut vitaminique D. C'est la 25OHD, représentant le stock de vitamine $\mathrm{D}$ de l'organisme, qui doit être dosée pour savoir si un patient a ou non une insuffisance en vitamine D.

Alors qu'il est maintenant consensuel que le statut vitaminique $\mathrm{D}$ doit être évalué par la mesure de la $25 \mathrm{OHD}$ et non par la mesure de la 1,25 (OH)2D, de nombreux experts ont discuté le fait que les valeurs de référence de 25OHD sont inadaptées (trop basses) et que les supplémentations recommandées sont insuffisantes [57-60], ce qui a amené une nouvelle approche pour l'établissement de ces valeurs de référence.

Lorsqu'on établit les valeurs de référence pour une variable biologique, on la mesure en général chez un grand nombre de sujets en bonne santé, représentatifs de la population de référence et on calcule un intervalle de référence (correspondant par exemple à $95 \%$ de la population). Si on pratique de cette façon pour la $25 \mathrm{OHD}$, et même en supposant que la même technique de dosage soit utilisée partout, les valeurs de référence dépendront de la population étudiée et en particulier de la saison pendant laquelle les échantillons sanguins ont été recueillis, de la latitude et de l'altitude du lieu où les sujets résident, ainsi que de leur âge et de la pigmentation de leur peau. La limite inférieure obtenue varie ainsi de $10 \mathrm{nmol} / \mathrm{L}$ (4 ng/mL) par exemple chez des sujets de race noire, prélevés en hiver et vivant à des latitudes supérieures à $40^{\circ}$, à 50 ou même $75 \mathrm{nmol} / \mathrm{L}$ ( $20 \mathrm{ou} 30 \mathrm{ng} / \mathrm{mL}$ ) pour des blancs ayant vécu pendant une période prolongée sous un ensoleillement intense. Même s'il est intéressant de connaître ces valeurs « usuelles », il ne faut surtout pas perdre de vue que le médecin qui prescrit un dosage de 25OHD veut dans l'immense majorité des cas tout 
simplement savoir si son patient ou sa patiente a une valeur normale ou basse pour, le cas échéant, lui prescrire de la vitamine $\mathrm{D}$. La fiabilité des valeurs de référence de 250HD (c'est-à-dire les valeurs auxquelles on doit se référer) a été mise en question par Heaney (entre autres) qui, en 2000, écrivait dans un éditorial [60] : «Lorsqu'il prescrit et interprète un dosage de vitamine $\mathrm{D}$, le médecin doit dans virtuellement tous les cas ignorer les normes publiées par le laboratoire... ». Lips [61], propose de définir l'insuffisance en vitamine D par des concentrations de 25OHD pour lesquelles il peut exister des effets délétères pour la santé et en particulier pour l'os (hyperparathyroïdie secondaire, augmentation du niveau de remodelage osseux). Cependant, ce concept est inhabituel pour les biologistes et la valeur seuil définissant l'insuffisance en vitamine D n'est pas facile à déterminer. Rappelons par ailleurs, que cette valeur (si elle existe) dépend du dosage utilisé et devrait être déterminée pour toutes les techniques de dosage (ce qui n'est pas le cas) [62-64]. Voici les différentes approches utilisées pour définir les concentrations de 25OHD associées à un statut vitaminique D optimal et par conséquent pour définir l'insuffisance en vitamine D. On peut les séparer en plusieurs categories.

\section{1. Étude de la relation entre les concentrations sériques de 25OHD et de PTH (ou entre les variations des concentrations de PTH et celles des concentrations de 25OHD) dans des populations apparemment en bonne santé}

Il s'agit ici de définir la concentration de 25OHD au-dessous de laquelle il peut exister une hyperparathyroïdie secondaire. Par hyperparathyroïdie secondaire, nous entendons une augmentation de la sécrétion de PTH afin de compenser une tendance à la baisse de la concentration sérique en calcium ionisé. Il n'est donc pas nécessaire d'avoir une PTH sérique au-dessus de la limite supérieure de la normale pour avoir une hyperparathyroïdie secondaire (d'autant plus que, pour une technique de dosage de PTH donnée, les valeurs de référence peuvent être significativement différentes en fonction de la population de référence recrutée [65]). Un sujet ayant par exemple une concentration sérique de PTH à $40 \mathrm{pg} / \mathrm{mL}$ (pour des valeurs de référence allant de 10 à $65 \mathrm{pg} / \mathrm{mL}$ ) mais dont la concentration de PTH en condition de balance calcique nulle est de $20 \mathrm{pg} / \mathrm{mL}$ peut être considéré comme ayant une hyperparathyroïdie suivant la définition proposée ci-dessus. Pour se convaincre du fait que la « normalité » de la PTH sérique n'exclut pas de possibles effets délétères, on peut se référer au fait que de très nombreux cas d'hyperparathyroïdies primitives avec PTH dite «normale » (c'est-à-dire dans la fourchette des valeurs de référence de la technique), confirmées par exérèse d'un adénome parathyroïdien ont été rapportés dans la littérature [66].

Dans cette optique, Dawson-Hughes et al. ont constaté des variations saisonnières de la PTH inversement liées aux variations de $25 \mathrm{OHD}$ chez des sujets de plus de 65 ans (182 hommes et 209 femmes) de la région de Boston. Ils ont constaté que la PTH pouvait augmenter dès que la $250 \mathrm{HD}$ sérique diminuait au-dessous de $44 \mathrm{ng} / \mathrm{mL}$ (110 nmol/L) [67]. Dans cette étude, les apports alimentaires moyens en calcium étaient de $730 \mathrm{mg} / \mathrm{j}$ environ.

Malabanan et al. ont donné de la vitamine D2 (50000 U par semaine pendant huit semaines, soit $400000 \mathrm{U}$ sur une période de 2 mois) et du calcium (1000-1500 $\mathrm{mg} / \mathrm{j})$ à 35 patients âgés de 49 à 83 ans dont la 250HD sérique de base était comprise entre 10 et $25 \mathrm{ng} / \mathrm{mL}(25-62,5 \mathrm{nmol} / \mathrm{L})$ [68]. Après ces huit semaines, la PTH sérique avait diminué significativement chez les sujets dont la $25 \mathrm{OHD}$ de base était inférieure à $20 \mathrm{ng} / \mathrm{mL}$ mais pas chez ceux $(n=7)$ dont la 25OHD était supérieure à $20 \mathrm{ng} / \mathrm{mL}$. Les auteurs ont conclu qu'une $25 \mathrm{OHD}$ sérique supérieure à $20 \mathrm{ng} / \mathrm{mL}$ au moins était nécessaire pour obtenir un concentration de PTH optimale.

De nombreuses études ont évalué la relation entre les concentrations de $25 \mathrm{OHD}$ et les concentrations de PTH dans des populations apparemment en bonne santé en cherchant à définir la concentration de 25OHD au-dessous de laquelle la PTH peut augmenter. L'immense majorité de ces études a constaté une relation statistique négative entre ces deux paramètres (la concentration de PTH baisse lorsque celle de 25OHD augmente). Cette relation n'était toutefois en général pas linéaire : la PTH diminuait jusqu'à ce que la $250 H D$ atteigne une valeur seuil au-dessus de laquelle la PTH est « en plateau ». Ces études ont été récemment listées et commentées par Aloia et al. [69]. Les valeurs seuil rapportées variaient grossièrement entre 16 et $44 \mathrm{ng} / \mathrm{mL}$ (40 et $110 \mathrm{nmol} / \mathrm{L}$ ) en fonction du mode de calcul utilisé pour quantifier la relation entre la 25OHD et la PTH (certains auteurs n'ont d'ailleurs pas trouvé de seuil de 25OHD au-dessus duquel la PTH ne diminuait plus) mais aussi en fonction de la population étudiée. Les apports calciques moyens de ces populations semblaient être un élément important. Schématiquement, la valeur seuil de $25 \mathrm{OHD}$ était aux alentours de $20 \mathrm{ng} / \mathrm{mL}$ ( $50 \mathrm{nmol} / \mathrm{L}$ ) lorsque les apports calciques moyens étaient de l'ordre de 1200$1500 \mathrm{mg} / \mathrm{j}$ et de $30-32 \mathrm{ng} / \mathrm{mL}(75-80 \mathrm{nmol} / \mathrm{L})$ lorsque les apports calciques étaient de l'ordre de 700-1000 mg/j). À noter que la relation entre les concentrations de PTH et les concentrations de $25 \mathrm{OHD}$ est probablement dépendante du statut en magnésium des sujets testés, une hypomagnésémie relative pouvant expliquer une non-élévation de la PTH malgré une 25OHD franchement basse [70].

\section{2. Évaluation des concentrations de $250 H D$ pour lesquelles l'absorption intestinale du calcium est optimale}

L'évaluation de l'absorption intestinale du calcium n'est pas facile et les études sont peu nombreuses. Elles suggèrent que l'absorption intestinale du calcium augmente lorsque la $25 \mathrm{OHD}$ augmente de 30 à $80-85 \mathrm{nM}(12$ à $32-34 \mathrm{ng} / \mathrm{mL})$ environ, puis qu'elle n'est plus significativement modifiée audelà [71].

\section{3. Étude de la relation entre les concentrations de $250 H D$ et la fréquence de certaines maladies}

Si tout le monde est d'accord pour considérer qu'il n'y a pas de rachitisme/ostéomalacie (les maladies associées à une carence 
profonde en vitamine D) lorsque la $25 \mathrm{OHD}$ est supérieure à 12 $25 \mathrm{nM}(5-10 \mathrm{ng} / \mathrm{mL})$, de très nombreuses études d'observation suggèrent une relation entre concentration basse de 25OHD et fréquence de nombreuses maladies (cancers, diabète, sclérose en plaques, polyarthrite rhumatoïde, tuberculose, HTA, « évènements » cardiovasculaires...) ou anomalies (sarcopénie...). En général, les sujets dans le quantile (tertile, quartile, quintile,..., décile) supérieur de 25OHD (concentrations en général $>75 \mathrm{nM}$ soit $30 \mathrm{ng} / \mathrm{mL}$ ) ont un risque relatif inférieur à ceux dans le quantile inférieur (par exemple [48,7274], mais aussi de très nombreux autres travaux).

Notons toutefois que ces études d'observation, même irréprochables en terme de méthodologie, ont une place inférieure aux études d'intervention dans la hiérarchie de l' « evidence-based medicine ».

\subsection{L'étude des concentrations moyennes de 25OHD atteintes (quand on les a !) dans les études d'intervention}

L'étude des concentrations moyennes de 25OHD atteintes (quand on les a !) dans les études d'intervention qui ont montré des effets positifs de la vitamine D sur :

- la réduction du risque de fractures (75 à $100 \mathrm{nM}$ soit 30 à $40 \mathrm{ng} / \mathrm{mL}$ ) [7] ;

- la réduction du risque de chutes (61-90 nM soit 24,4-36 ng/ $\mathrm{mL})$ [19] ;

- la réduction du risque de cancer (on se base sur une étude pour laquelle la 25OHD moyenne atteinte était de $96 \mathrm{nM}$ $(38,4 \mathrm{ng} / \mathrm{mL})$ à partir d'une valeur de base de $71 \mathrm{nM}(28,4 \mathrm{ng} /$ mL) [33]) ;

- la réduction du risque de chute des dents (là encore, une seule étude où les concentrations de $25 \mathrm{OHD}$ sont montées de 71 à $110 \mathrm{nM}$ soit $28,4-44 \mathrm{ng} / \mathrm{mL}$ [75]) ;

- la réduction de la pression artérielle après trois mois d'exposition à des UVB pendant lesquels la 25OHD est passée de 58 à $151 \mathrm{nmol} / \mathrm{L}$ (23,2 à 60,4 ng/mL) [54], ou après $1200 \mathrm{mg}$ de calcium et $800 \mathrm{UI} / \mathrm{J}$ de vitamine D3 pendant deux mois pendant lesquels la 25OHD est passée de 25,7 à 64,8 nM $(10,3$ à 25,9 ng/mL) [12].

Les résultats (même variés) de ces différentes approches sont cohérents avec la proposition faite en 2005 par cinq des six experts réunis dans une table ronde, de définir l'insuffisance en vitamine $\mathrm{D}$ par des concentrations de $25 \mathrm{OHD}$ inférieures à $75 \mathrm{nM}(30 \mathrm{ng} / \mathrm{mL})$ «... it is important to ensure that the serum $25 O H D$ level obtained after vitamin $D$ supplementation in individual patients reaches this new threshold. » [76].

Les deux conséquences principales de cette nouvelle définition du seuil d'insuffisance en vitamine D sont :

- l'insuffisance en vitamine $\mathrm{D}$ est très fréquente : à des latitudes $>40^{\circ}$ (l'Europe par exemple) et suivant les populations testées et la période de l'année, 50 à presque $100 \%$ des individus ne recevant pas de supplémentation ont une concentration sérique de $250 \mathrm{HD}$ inférieure à $30 \mathrm{ng} / \mathrm{mL}$ [77-84] (et Tableau 1) ;

- les apports nutritionnels conseillés (ANC) pour la vitamine D (400 U/J pour les sujets de moins de 65 ans et $600 \mathrm{U} / \mathrm{J}$ pour ceux de plus de 65 ans) sont insuffisants pour atteindre cette cible minimale de $30 \mathrm{ng} / \mathrm{mL}$ de $250 \mathrm{HD}$ [85] et il faut donc envisager des doses plus importantes. Il est légitime alors de se poser la question d'un éventuel surdosage. La vitamine D est en effet potentiellement toxique. Un excès de vitamine $\mathrm{D}$ a pour conséquence une augmentation de l'absorption intestinale du calcium. La tendance hypercalcémique qui en résulte freine la sécrétion de PTH ce qui augmentera la calciurie avec des risques rénaux potentiels (lithiase, néphrocalcinose). L'étape suivante sera caractérisée par une hypercalcémie, parfois très sévère, surtout si la fonction rénale est altérée. Cette situation est en fait très rare. Les différentes revues de la littérature concernant les cas

Tableau 1

Prévalence de l'insuffisance en vitamine D (concentration sérique de $25 \mathrm{OHD}<30 \mathrm{ng} / \mathrm{mL}$, soit 75 nmol/L, mesurée avec le dosage RIA DiaSorin ou exprimée en « équivalent RIA DiaSorin ») dans quelques études françaises et européennes.

Prevalence of the vitamin D insufficiency (serum 25 OHD levels $<30 \mathrm{ng} / \mathrm{ml}$ or $75 \mathrm{nmol} / \mathrm{L}$ measured with the Diasorin RIA or expressed as "Diasorin RIA equivalent") in some European and French studies.

\begin{tabular}{|c|c|c|c|}
\hline Référence & Population & Lieu & $\begin{array}{l}\text { Pourcentage } \\
\text { d'insuffisances en } \\
\text { vitamine D }(25 \mathrm{OHD} \\
<30 \mathrm{ng} / \mathrm{mL})(\%)\end{array}$ \\
\hline $\begin{array}{l}\text { Étude DHEage Souberbielle JC et al. } \\
\text { J Clin Endocrinol Metab } 2001 \text {; 86:3086-3090. }\end{array}$ & $\begin{array}{l}280 \text { sujets en bonne santé }(140 \mathrm{H}, 140 \mathrm{~F}) \text {, } \\
60-79 \text { ans }\end{array}$ & France région parisienne & 88 \\
\hline $\begin{array}{l}\text { Étude SUVIMAX Chapuy MC et al. Osteoporos Int } \\
\qquad 1997 ; 7: 439-443\end{array}$ & $\begin{array}{l}1579 \text { sujets en bonne santé }(805 \mathrm{~F}, 774 \mathrm{H}) \\
35-60 \text { ans }\end{array}$ & France toutes régions & 78 \\
\hline Fardellonne P et al. Rev Rhum Engl Ed 1995 ; 62:576-581 & $\begin{array}{l}126 \text { patients institutionnalisés ( } 99 \mathrm{~F}, 27 \mathrm{H}) \text {, } \\
\text { âge moyen de } 84 \text { ans }\end{array}$ & $\begin{array}{l}\text { France (Picardie, région } \\
\text { parisienne, Montpellier) }\end{array}$ & 100 \\
\hline \multirow[t]{6}{*}{ Lips P et al. J Intern Med 2006; 260:245-254. } & $\begin{array}{l}2606 \text { femmes ménopausées ostéoporotiques } \\
\text { (âge moyen : } 68 \text { ans) }\end{array}$ & France & 49,7 \\
\hline & & Suisse & 63,3 \\
\hline & & Pays-bas & 52,0 \\
\hline & & Royaume-Uni & 74,5 \\
\hline & & Allemagne & 68,0 \\
\hline & & Espagne & 64,7 \\
\hline
\end{tabular}


d'intoxications à la vitamine D [86] suggèrent qu'elle n'apparaît jamais pour des concentrations de 25OHD inférieures à $375 \mathrm{nmol} / \mathrm{L}(150 \mathrm{ng} / \mathrm{mL})$. Plusieurs études ont par ailleurs démontré que des doses journalières de vitamine D largement supérieures aux ANC (plus de $4000 \mathrm{UI} / \mathrm{J}$ ) sont sans conséquence sur la calciurie ou la calcémie $[2,87,88]$.

Les valeurs de référence de la 25OHD doivent donc définir le statut vitaminique D optimal. Dans nos laboratoires, nous les appelons valeurs « souhaitables ». Elles sont de 30 à $80 \mathrm{ng} / \mathrm{mL}$ (soit 75 à $200 \mathrm{nmol} / \mathrm{L}$ ). La limite supérieure a été choisie arbitrairement pour être suffisamment éloignée de la zone de toxicité potentielle. Étant attentif à la littérature scientifique (abondante), nous n'excluons pas que ces valeurs souhaitables puissent « évoluer » en fonction des données publiées. Par ailleurs, nous n'indiquons (surtout) pas sur nos feuilles de résultats les valeurs retrouvées dans une population apparemment en bonne santé car cela peut être très perturbant pour le clinicien prescripteur.

\section{Correction d'une insuffisance en vitamine $D$ et maintien d'un statut vitaminique $D$ optimal}

Il s'agit de deux actions différentes qui souvent nécessiteront d'utiliser des doses différentes. Une question importante est de savoir si on doit doser la 25OHD avant de donner une supplémentation en vitamine D. Plus précisément, connaît-on la dose de vitamine $\mathrm{D}$ qui permettrait de monter la concentration de $25 \mathrm{OHD}$ de $95 \%$ de la population au-dessus de $30 \mathrm{ng} / \mathrm{mL}$ (même chez ceux qui ont une concentration de 25OHD initiale $<5 \mathrm{ng} / \mathrm{mL}$ ) sans que personne ou presque n' ait une concentration supérieure à $100 \mathrm{ng} / \mathrm{mL}$ (même ceux qui au départ ont $50 \mathrm{ng} / \mathrm{mL}$ ou plus). Il n'y a pas aujourd'hui de réponse claire à cette question et il faut prendre en compte la très grande variabilité individuelle de l'augmentation de la concentration de 25OHD en réponse à une dose donnée de vitamine D. Des protocoles sont en cours et d'autres sont prévus pour essayer d'y répondre. On peut toutefois signaler une étude récente [89] chez 138 sujets qui ont reçu pendant six mois des doses journalières de vitamine $\mathrm{D}$ avec deux réajustements de posologie en fonction des concentrations de 25OHD mesurées sous traitement. Au bout de six mois, quasiment tous les sujets avaient une concentration de 250HD supérieure à $75 \mathrm{nmol} / \mathrm{L}$ ( $30 \mathrm{ng} / \mathrm{mL})$ et aucun n'avait une concentration supérieure à $220 \mathrm{nmol} / \mathrm{L}(88 \mathrm{ng} / \mathrm{mL})$. Aucun cas d'hypercalcémie ni d'hypercalciurie n'a été observé. La conclusion des auteurs était que, pour obtenir une concentration de 250HD entre 75 et $220 \mathrm{nmol} / \mathrm{L}$, il faut donner 3800 UI de vitamine D3 par jour si la concentration de base de $25 \mathrm{OHD}$ est supérieure à $55 \mathrm{nmol} / \mathrm{L}$ (22 ng/mL) et 5000 UI par jour si la concentration de base est inférieure à $55 \mathrm{nmol} / \mathrm{L}$ (et donc qu'il faut doser la 25OHD).

Aujourd'hui, nous dosons la 25OHD et nous donnons des doses de vitamine D qui dépendent de la valeur mesurée. Nous nous basons en partie sur les propositions de Holick dans sa revue récente [1]. Notre pratique (résumée dans le Tableau 2) est de donner quatre ampoules de 100000 UI de vitamine D3 (1 ampoule toutes les 2 semaines) si la 25OHD est inférieure ou égale à $10 \mathrm{ng} / \mathrm{mL}$, trois ampoules si la $25 \mathrm{OHD}$ est entre 11 et $20 \mathrm{ng} / \mathrm{mL}$ et deux ampoules si la $25 \mathrm{OHD}$ est entre 21 et $30 \mathrm{ng} / \mathrm{mL}$.

Tableau 2

Exemple de protocole de correction d'une insuffisance en vitamine D (puis de prescription d'un traitement «d'entretien ») en fonction de la concentration sérique de 25OHD (ce protocole est basé en partie sur les propositions de Holick [1]).

How to treat a vitamin D insufficiency and to maintain a sufficient level of 25-OHD, in function of the initial serum 25-OHD levels of the patient (partially inspired from Holick's proposals [1]).

Étape 1:

Correction d'une insuffisance en vitamine D (c'est-à-dire donner de la vitamine D afin de faire monter la concentration sérique de $25 \mathrm{OHD}$ au-dessus de $30 \mathrm{ng} / \mathrm{mL}$, soit $75 \mathrm{nmol} / \mathrm{L}$ )

Si la concentration sérique de $25 \mathrm{OHD}$ est $<10 \mathrm{ng} / \mathrm{mL}$ prescrire une ampoule de 100000 UI de vitamine D3 toutes les deux semaines pendant 2 mois (soit 4 ampoules au total)

Si la concentration sérique de 25OHD est $10-20 \mathrm{ng} / \mathrm{mL}$ prescrire une ampoule de 100000 UI de vitamine D3 toutes les deux semaines pendant 1,5 mois (soit 3 ampoules au total)

Si la concentration sérique de $25 \mathrm{OHD}$ est $>20 \mathrm{ng} / \mathrm{mL}$ mais $<30 \mathrm{ng} / \mathrm{mL}$ prescrire une ampoule de 100000 UI de vitamine D3 toutes les deux semaines pendant 1 mois (soit 2 ampoules au total)

Étape 2 (qui suppose que l'étape 1 a été effectuée) :

Maintien d'une concentration sérique de $25 \mathrm{OHD}>30 \mathrm{ng} / \mathrm{mL}$. Il faut privilégier l'observance. En fonction des préférences des patients, on peut prescrire :

Des doses « espacées » de vitamine D3 (100000 UI de vitamine D3 tous les 2 ou 3 mois par exemple)

Des doses journalières (800 à 1200 UI selon les experts mais ces recommandations sont susceptibles d'évoluer) de vitamine D3 (en France les spécialités permettant ces doses journalières de vitamine D3 sont des associations calcium + vitamine D3 et il faut donc préalablement évaluer les apports calciques alimentaires pour ne donner ces spécialités que si les apports calciques sont insuffisants) ou de vitamine D2 (lorsqu'on ne veut pas associer la vitamine $\mathrm{D}$ à du calcium ou lorsque la supplémentation en calcium médicamenteux ne nécessite que $500 \mathrm{mg}$ )

Signalons par ailleurs les associations bisphosphonate/vitamine D3 (5600 UI de vitamine D3 par semaine, soit l'équivalent de 800 UI/jour) prescrites aujourd'hui fréquemment chez les patients ostéoporotiques.

La vérification de l'efficacité du traitement correcteur ou de maintien peut se faire par le dosage de la 25OHD sérique.

Pour vérifier l'efficacité du traitement correcteur (étape 1), doser la 25OHD environ une semaine après la dernière ampoule de vitamine D3

Pour vérifier si la fréquence des doses « espacées » du traitement d'entretien (étape 2) est adaptée, doser la $25 \mathrm{OHD}$ juste avant la prise d'une ampoule (par exemple si on prescrit une ampoule tous les 3 mois, la constatation d'une $25 \mathrm{OHD}<30 \mathrm{ng} / \mathrm{mL}$ juste avant la prise incitera à prescrire plutôt une ampoule tous les 2 mois)

-Pour vérifier si la posologie de doses journalières est suffisante, doser la 25OHD après 3-4 mois de traitement journalier (ou hebdomadaire en cas de traitement par association bisphosphonate/vitamine D3). En cas de traitement par vitamine D2, bien s'assurer auprès du laboratoire que la technique de dosage de 25OHD utilisée mesure bien la 25OHD2 et la 25OHD3 
Pour maintenir ensuite une concentration supérieure à $30 \mathrm{ng} /$ $\mathrm{mL}$ on peut donner soit des doses journalières (800 à $1200 \mathrm{UI} / \mathrm{J}$ de vitamine D2 ou de vitamine D3) ou bien une ampoule de 100000 UI de vitamine D3 tous les deux mois (le choix dépendra de la préférence des patients, le but étant de privilégier l'observance).

Vous remarquerez que lorsque nous donnons des doses «ponctuelles », nous utilisons aujourd'hui de la vitamine D3 plutôt que de la vitamine D2. Nous nous basons pour cela sur des études qui ont montré que, pour une même dose administrée, la vitamine D 3 permettait de maintenir la 25OHD élevée plus longtemps que la vitamine D2 [90-92]. Nous devons toutefois signaler que cela n'a pas été retrouvé par toutes les équipes [93]. En revanche, l'administration de doses journalières peut a priori se faire indifféremment avec la vitamine D3 ou la vitamine D2.

\section{Conclusion}

Les connaissances sur la vitamine D ont beaucoup progressé ces dernières années et de nombreuses études sont en cours comme en témoigne le nombre très important de publications sur le sujet. La vitamine $\mathrm{D}$ ne peut plus être considérée comme étant uniquement nécessaire à la prévention des rachitismes/ostéomalacies. Son rôle dans la prévention des fractures ostéoporotiques périphériques du sujet âgé est maintenant bien démontré (en association avec du calcium) et de très nombreuses données épidémiologiques et expérimentales sont en faveur d'un rôle dans la prévention de nombreuses affections (certains cancers et maladies auto-immunes, évènements cardiovasculaires et hypertension, sarcopénie du sujet âgé...). Il existe même quelques études d'intervention confirmant certains de ces effets et une méta-analyse récente de 18 essais randomisés (plus de 57000 patients) a conclu à une diminution du risque de mortalité chez les sujets ayant un apport régulier de vitamine D [94]. L'évaluation du statut vitaminique $\mathrm{D}$ peut être aisément réalisée par le dosage de la 25OHD sérique. Toutefois, la plupart des revues récentes sur le sujet suggèrent que les valeurs de référence de la 25OHD obtenues dans des populations apparemment en bonne santé sont beaucoup trop basses et que la concentration de 25OHD au-dessous de laquelle il existe une insuffisance en vitamine D se situe entre 50 et $100 \mathrm{nmol} / \mathrm{L}$ ( 20 et $40 \mathrm{ng} / \mathrm{mL}$ ) avec une franche tendance à désirer des valeurs supérieures à $75 \mathrm{nmol} /$ L (30 ng/mL). Dans ce contexte, les supplémentations habituellement recommandées sont insuffisantes pour atteindre ces concentrations.

\section{Conflits d'intérêts}

Aucun

\section{Références}

[1] Holick M. Vitamin D deficiency. N Engl J Med 2007;357:266-81.

[2] Heaney R, Davies M, Chen T, Holick M, Barger-Lux J. Human serum 25hydroxycholecalciferol response to extended oral dosing with cholecalciferol. Am J Clin Nutr 2003;77:204-10.
[3] Nykjaer A, Dragun D, Walther D, Vorum H, Jacobsen C, Herz J, et al. An endocytic pathway essential for renal uptake and activation of the steroid 25-(OH) vitamin D3. Cell 1999;96:507-15.

[4] Hoenderop J, Nilius B, Bindels R. Calcium absorption across epithelia. Physiol Rev 2005;85:373-422.

[5] White KE, Biber J, Murer H, Econs MJ. Chromosomal localization of two human genes involved in phosphate homeostasis: the type IIb sodiumphosphate cotransporter and stanniocalcin-2. Somat Cell Mol Genet 1998; 24:357-62.

[6] Basha B, Rao S, Han ZH, Parfitt M. Osteomalacia due to vitamin D depletion: a neglected consequence of intestinal malabsorption. Am J Med 2000;108:296-300.

[7] Bischoff-Ferrari, Willett W, Wong JB, Giovanucci E, Dietrich T, DawsonHughes B. Fracture prevention with vitamin D supplementation: a metaanalysis of randomized controlled trials. JAMA 2005;293:2257-64.

[8] Tang B, Eslick GD, Nowson C, Smith C, Bensoussan A. Use of calcium in combination with vitamin $\mathrm{D}$ supplementation to prevent fractures and bone loss in people aged 50 years and older: a meta-analysis. Lancet 2007;370: 657-66.

[9] Van der Mei I, Ponsonby AL, Dwyer T, Blizzard L, Simmons R, Taylor B, et al. Past exposure to sun, skin phenotype, and risk of multiple sclerosis : case-control study. BMJ 2003;327:316-21.

[10] Hanchette C, Schwartz G. Geographic patterns of prostate cancer mortality. Evidence for a protective effect of ultraviolet radiation. Cancer 1992;70:2861-9.

[11] Garland CF, Garland FC, Gorham ED, Young JF. Geographic variation in breast cancer mortality in the United States: a hypothesis involving exposure to solar radiation. Prev Med 1990;19:614-22.

[12] Pfeifer M, Begerow B, Minne H, Nachtigall D, Hansen C. Effects of a short-term vitamin D3 and calcium supplementation on blood pressure and parathyroid hormone levels in elderly women. J Clin Endocrinol Metab 2001;86:1633-7.

[13] Zittermann A, Schultze Schleithoff S, Tenderich G, Berthold H, Körfer R, Stehle P. Low vitamin D status: a contributing factor in the pathogenesis of congestive heart failure? J Am Coll Cardiol 2003;41:105-12.

[14] McKenna M, Freaney R. Secondary hyperparathyroidism in the elderly: means to defining hypovitaminosis D.. Osteoporos Int 1998;8(suppl 1):S3-6.

[15] Eastwood J, de Wardener H, Gray R, Leman J. Normal plasma 1,25(OH)2 vitamin D in nutritional osteomalacia. Lancet 1979: 1377-1378.

[16] Bouillon R, Bischoff-Ferrari H, Willett W. Vitamin D and health: perspective from mice and man. J Bone Miner Res 2008. [Epub ahead of print].

[17] Visser M, Deeg D, Lips P. Low vitamin D and high parathyroid hormone levels as determinants of loss of muscle strength and muscle mass (sarcopenia): the longitudinal aging study Amsterdam. J Clin Endocrinol Metab 2003;88:5766-72.

[18] Zhu K, Dick I, Devine A, Bruce D, Prince R. An RCT of vitamin D or placebo on falls in elderly women with low vitamin D status and a falling history. J Bone Miner Res 2006;21:1227.

[19] Bischoff-Ferrari, Dawson-Hughes B, Willet W, Staehelin H, Bazemore M, Zee R, et al. Effect of vitamin D on falls. A meta-analysis. JAMA 2004;291:1999-2006.

[20] Sato Y, Iwamoto J, Kanoko T, Satoh K. Low-dose vitamin D prevents muscular atrophy and reduces falls ans hip fractures in women after stroke: a randomized controlled trial. Cerebrovasc Dis 2005;20:187-92.

[21] Capiati DA, Vasquez G, Boland RL. Protein kinase C alpha modulates the $\mathrm{Ca} 2+$ influx phase of the $\mathrm{Ca} 2+$ response to 1 alpha, 25-dihydroxy-vitaminD3 in skeletal muscle cells. Horm metab res 2001;33:201-6.

[22] Garland FC, Garland CF. Do sunlight and vitamin D reduce the likelihood of colon cancer? Int J Epidemiol 1980;9:227-31.

[23] Grant WB. An estimate of premature cancer mortality in the US due to inadequate doses of solar ultraviolet-B-radiation. Cancer 2002;94:1867-75.

[24] Mizoue T. Ecological study of solar radiation and cancer mortality in Japan. Health Phys 2004;87:532-8.

[25] Garland CF, Comstock G, Garland FC, Helsing K, Shaw E, Gorham E. Serum 25 hydroxy vitamin D and colon cancer: eight year prospective study. Lancet 1989;334:1176-8. 
[26] Giovannucci E, Liu Y, Rimm E, Hollis B, Fuchs C, Stampfer M, et al. Prospective study of predictors of vitamin D status and cancer incidence and mortality in men. J Natl Cancer Inst 2006;98:451-9.

[27] Bostick R, Potter J, Sellers T, McKenzie D, Kushi L, Folsom A. Relation of calcium, vitamin D, and dairy food intake to incidence of colon cancer among older women. Am J Epidemiol 1993;137:1302-17.

[28] Feskanich D, Ma J, Fuchs CS, Kirkner GJ, Hankinson SE, Hollis BW, et al. Plasma vitamin D metabolites and risk of colorectal cancer in women. Cancer Epidemiol Biomarkers Prev 2004;13:1502-8.

[29] Ahonen M, Tenkanen L, Teppo L, Hakama M, Tuohlmaa P. Prostate cancer risk and prediagnostic serum 25 -hydroxyvitamin D levels. Cancer Causes and Control 2000;11:847-52.

[30] Skinner H, Michaud D, Giovanucci E, Willett W, Colditz G, Fuchs C. Vitamin D intake and the risk for pancreatic cancer in two cohort studies. Cancer Epidemiol Biomarkers Prev 2006;15:1688-95.

[31] Zhou W, Heist R, Liu G, Asomaning K, Neuberg D, Hollis B, et al. Circulating 25-hydroxyvitamin D levels predict survival in earlystage non-small-cell lung cancer patients. J Clin Oncol 2007;25: $479-85$.

[32] Wactawski-Wende J, Kotchen JM, Anderson GL, Assaf AR, Brunner RL, O'Sullivan MJ, et al. Calcium plus vitamin D supplementation and the risk of colorectal cancer. N Engl J med 2006;354:684-96.

[33] Lappe JM, Travers-Gustafson D, Davies KM, Recker R, Heaney R. Vitamin D and calcium supplementation reduces cancer risk: results of a randomized trial. Am J Clin Nutr 2007;85:1586-91.

[34] Carlberg C. Current understanding of the function of the nuclear vitamin $D$ receptor in response to its natural and synthetic ligands. Recent Results Cancer Res 2003;164:29-42.

[35] Tangpricha V, Spina C, Yao M, Chen TC, Wolfe MM, Holick M. Vitamin D deficiency enhances the growth of MC-26 colon cancer xenografts in Balb/c mice. J Nutr 2005;135:2350-4.

[36] Mantel DJ, Owens NJ, Bundred EB, Mawer AE, Canfield AE. 1,25dihydroxyvitamin D3 inhibits angiogenesis in vitro and in vivo. Circ Res 2000;87:214-20.

[37] Simboli-Campbell M, Narvaez CJ, Tenniswood M, Welsh J. 1,25-dihydroxyvitamin D3 induces morphological and biochemical markers of apoptosis in MCF-7 breast cancer cells. J Steroid Biochem Mol Biol 1996;58:367-76.

[38] Shabahang M, Buras RR, Davoodi F, Shumaker LM, Nauta RJ, Uskokovic MR, et al. Growth inhibition of HT-29 human colon cancer cells by analogues of 1,25-dihydroxyvitamin D3. Cancer Res 1994;54:4057-64.

[39] Saggese G, Frederico G, Balestri M, Torriolo A. Calcitriol inhibits the PHA-induced production of IL-2 and IFN-gamma and the proliferation of human peripheral blood leucocytes while enhancing the surface expression of HLA class II molecules. J Clin Invest 1989;12:329-35.

[40] Cantorna M, Hayes C, DeLuca H. 1,25-dihydroxyvitamin D D3 reversibly blocks the progression of relapsing encephalomyelitis, a model of multiple sclerosis. Proc Natl Acad Sci USA 1996;93:7861-4.

[41] Lemire JM, Ince A, Takashima M. 1,25-dihydroxyvitamin D3 attenuates the expression of experimental murine lupus of MRL/1 mice. Autoimmunity 1992;12:143-8.

[42] Cantorna M, Hayes C, DeLuca H. 1,25-dihydroxycholecalciferol inhibits the progression of arthritis in murine models of human arthritis. J Nutr 1998;128:68-72.

[43] Mathieu C, Waer M, Laureys J, Rutgeerts O, Bouillon R. Prevention of type I diabetes in NOD mice by 1,25-dihydroxyvitamin D3. Diabetologia 1994;37:552-8.

[44] Cantorna M, Woodward W, Hayes C, DeLuca HY. 1,25-dihydroxyvitamin D3 is a positive regulator for the two anti-encephalitogenic cytokines TGFß1 and IL-4. J Imunol 1998;160:5314-9.

[45] DeLuca H, Cantorna M. Vitamin D: its role in immunology. FASEB J 2001;15:2579-85.

[46] Hyppönen E, Läära E, Reunanen A, Järvelin MR, Virtanen S. Intake of vitamin D and risk of type 1 diabetes: a birth-cohort study. Lancet 2001;358:1500-3.

[47] Stene L, Ulriksen J, Magnus P, Joner G. Use of cod liver oil during pregnancy associated with lower risk of type I diabetes in the offspring. Diabetologia 2000;43:1093-8.
[48] Munger KL, Levin LI, Hollis BW, Howard NS, Ascherio A. Serum 25hydroxyvitamin D levels and risk of multiple sclerosis. JAMA 2006;296: 2832-8.

[49] McAlindon T, Felson D, Zhang Y, Hannan M, Aliabadi P, Weissman B, et al. Relation of dietary intake and serum levels of vitamin $\mathrm{D}$ to progression of arthritis of the knee among participants in the Framingham study. Ann Int Med 1996;125:353-9.

[50] Liu PT, Stenger S, Li H, Wenzel L, Tan BH, Krutzik SR, et al. Toll-like receptor triggering of a vitamin D-mediated human antimicrobial response. Science 2006;311:1770-3.

[51] Chan TYK. Vitamin D deficiency and susceptibility to tuberculosis. Calcif Tissue Int 2000;66:476-8.

[52] Wang T, Pencina M, Booth S, Jacques P, Ingelsson E, Lanier K, et al. Vitamin D deficiency and risk of cardiovascular disease. Circulation 2008;117:503-11.

[53] Li YC, Kong J, Wei M, Chen ZF, Liu SQ, Cao LP. 1,25-dihydroxyvitamin $\mathrm{D}(3)$ is a negative endocrine regulator of the rennin-angiotensin system. J Clin Invest 2002;110:229-38.

[54] Krause R, Buhring M, Hopfenmuller W, Holick M, Sharma AM. Ultraviolet B and blood pressure. Lancet 1998;352:709-10.

[55] Baynes KCR, Boucher BJ, Feskens EJM, Kromhout D. Vitamin D, glucose tolerance and insulinaemia in elderly men. Diabetologia 1997; 40:344-7.

[56] Barger-Lux M, Heaney R, Lanspa S, Healy JC, DeLuca H. An investigation of sources of variation in calcium absorption efficiency. J Clin Endocrinol Metab 1995;80:406-11.

[57] Zittermann A. Vitamin D in preventive medicine: are we ignoring the evidence? British Journal of Nutrition 2003;89:552-72.

[58] Hollis B. Circulating 25-hydroxyvitamin D levels indicative of vitamin D sufficiency: implications for establishing a new effective dietary intake recommendation for vitamin D. J Nutr 2005;135:317-22.

[59] Holick M. Too little vitamin D in premenopausal women: why should we care? Am J Clin Nutr 2002;76:3-4.

[60] Heaney R. Vitamin D: how much do we need, and how much is too much? Osteoporos Int 2000;11:553-5.

[61] Lips P. Vitamin D deficiency and secondary hyperparathyroidism in the elderly: consequences for bone loss and fractures and therapeutic implications. Endocrine Reviews 2001;22:477-501.

[62] Lips P, Chapuy MC, Dawson-Hughes B, Pols H, Holick M. An international comparison of serum 25-hydroxy vitamin D measurements. Osteoporos Int 1999;9:394-7.

[63] Binkley N, Krueger D, Cowgill C, Plum L, Lake E, Hansen H, et al. Assay variation confounds the diagnosis of hypovitaminosis $\mathrm{D}$ : a call for standardization. J Clin Endocrinol Metab 2004;89:3152-7.

[64] Hollis B. Editorial: the determination of circulating 25-hydroxyvitamin D: no easy task. J Clin Endocrinol Metab 2004;89:3149-51.

[65] Souberbielle JC, Cormier C, Kindermans C, Gao P, Cantor T, Forette F, et al. Vitamin $\mathrm{D}$ status and redefining serum parathyroid hormone reference range. J Clin Endocrinol Metab 2001;86:3086-90.

[66] Glendenning P, Gutteridge DH, Retallack, Stuckey BG, kermode DG, Kent N. High prevalence of normal total calcium and intact PTH in 60 patients with proven primary hyperparathyroidism: a challenge to current diagnostic criteria. Aust NZ J Med 1998;28:173-8.

[67] Dawson-Hughes B, Harris S, Dallal G. Plasma calcidiol, season, and serum parathyroid hormone concentrations in healthy elderly men and women. Am J Clin Nutr 1997;65:67-71.

[68] Malabanan A, Veronikis IE, Holick M. Redefining vitamin D insufficiency. Lancet 1998;351:805-6.

[69] Aloia J, Talwar S, Pollack S, Feurman M, Yeh J. Optimal vitamin D status and serum parathyroid hormone concentrations in African American women. Am J Clin Nutr 2006;84:602-9.

[70] Sahota O, Mundey MK, San P, Godber IM, Hosking DJ. Vitamin D insufficiency and the blunted PTH response in established osteoporosis: the role of magnesium deficiency. Osteoporos Int 2006;17:101321.

[71] Heaney R, Dowell MS, Hale CA, Bendich A. Calcium absorption varies within the reference range for serum 25 -hydroxy vitamin D. J Am Coll Nutr 2003;22:142-6. 
[72] Garland CF, Gorham ED, Mohr SB, Grant WB, Giovanucci E, Lipkin M, et al. Vitamin D and prevention of breast cancer:pooled analysis. J Steroid Mol Biol 2007;103:708-11.

[73] Gorham ED, Garland CF, Garland FC, Grant WB, Mohr SB, Lipkin M, et al. Optimal vitamin D status for colorectal cancer prevention: a quantitative meta analysis. Am J Prev Med 2007;32:210-6.

[74] Forman J, Giovanucci E, Holmes MD, Bischoff-Ferrari HA, Tworoger SS, Willett WC, et al. Plasma 25-hydroxyvitamin D levels and risk of incident hypertension. Hypertension 2007;49:1063-9.

[75] Krall EA, Wehler C, Garcia RI, Harris SS, Dawson-Hughes B. Calcium and vitamin D supplements reduce tooth loss in the elderly. Am J Med 2001;111:452-6.

[76] Dawson-Hughes B, Heaney R, Holick M, Lips P, Meunier PJ, Vieth R. Estimates of optimal vitamin D status. Osteoporos Int 2005;16:713-6.

[77] Chapuy MC, Preziosi P, Maamer M, Arnaud S, Galan P, Hercberg S, et al. Prevalence of vitamin D insufficiency in an adult normal population. Osteoporos Int 1997;7:439-43.

[78] Kinyamu K, Gallagher C, Rafferty K, Balhorn K. Dietary calcium and vitamin $\mathrm{D}$ intake in elderly women: effect on serum parathyroid hormone and vitamin D metabolites. Am J Clin Nutr 1998;67:342-8.

[79] Thomas M, Lloyd-Jones D, Thadhani R, Shaw A, Deraska D, Kitch B, et al. Hypovitaminosis $\mathrm{D}$ in medical inpatients. $\mathrm{N}$ Engl $\mathrm{J}$ Med 1998;338:777-83.

[80] LeBoff M, Kohlmeier L, Hurwitz S, Franklin J, Wright J, Glowacki J. Occult vitamin D deficiency in postmenopausal US women with acute hip fracture. JAMA 1999;281:1505-11.

[81] Glerup H, Mikkelsen K, Poulsen L, Hass E, Overbeck S, Thomsen J, et al. Commonly recommended daily intake of vitamin D is not sufficient if sunlight exposure is limited. Journal of Internal Medicine 2000;247:260-8.

[82] Isaia G, Giorgino R, Rini GB, Bevilacqua M, Maugeri D, Adami S. Prevalence of hypovitaminosis D in elderly women in Italy; clinical consequence and risk factors. Osteoporosis Int 2003;14:577-82.

[83] Harris S, Soteriades E, Coolidge AS, Mudgal S, Dawson-Hughes B. Vitamin $\mathrm{D}$ insufficiency and hyperparathyroidism in a low income, multiracial, elderly population. J Clin Endocrinol Metab 2000;85:4125-30.

[84] Lips P, Duong T, Oleksik A, Black D, Cummings S, Cox D, et al. A global study of vitamin D status and parathyroid function in postmenopausal women with osteoporosis: baseline data from the multiple outcome of Raloxifene evaluation clinical trial. J Clin Endocrinol Metab 2001;86: 1212-21.

[85] Vieth R. Why the optimal requirement for vitamin D3 is probably much higher than what is officially recommended for adults. J Steroid Biochem Mol Biol 2004;89:575-9.

[86] Vieth R. Vitamin D supplementation, 25-hydroxyvitamin D concentrations, and safety. Am J Clin Nutr 1999;69:842-56.

[87] Vieth R, Chan PC, McFarlane G. Efficacy and safety of vitamin D3 intake exceeding the lowest observed adverse effect level. Am J Clin Nutr 2001; 73:288-329.

[88] Barger-Lux MJ, Heaney RP. Effects of above average summer sun exposure on serum 25-hydroxyvitamin D and calcium absorption. J Clin Endocrinol Metab 2002;87:4952-6.

[89] Aloia J, Patel M, Dimaano R, Li-Ng M, Talwar SA, Mickhail M, et al. Vitamin D intake to attain a desired serum 25-hydroxy vitamin D concentration. Am J Clin Nutr 2008;87:1952-8.

[90] Armas LAG, Hollis BW, Heaney RP. Vitamin D2 is much less effective than vitamin D3 in humans. J Clin Endocrinol Metab 2004;89:5387-91.

[91] Trang HM, Cole DEC, Rubin LA, Pierratos A, Siu S, Vieth R. Evidence that vitamin D3 increases serum 25-hydroxyvitamin D more efficiently than does vitamin D2. Am J Clin Nutr 1998;68:854-8.

[92] Romagnoli E, Mascia ML, Cipriani C, Fassino V, Mazzei F, D’Erasmo E, Carnevale V, Scillitani A, Minisola S. Short and long term variations in serum calciotropic hormones after a single very large dose of ergocalciferol (vitamin D2) or cholecalciferol (vitamin D3) in the elderly. J Clin Endocrinol Metab 2008 May 20.[Epub ahead of print].

[93] Holick M, Biancuzzo RM, Chen TC, et al. Vitamin D2 is as effective as vitamin D3 in maintaining circulating concentrations of 25-hydroxyvitamin D. J Clin Endocrinol Metab 2008;93:677-81.

[94] Autier P, Gandini S. Vitamin D supplementation and total mortality: a meta-analysis of randomized controlled trials. Arch Intern Med 2007;167: 1730-7.

[95] Segersten U, Correa P, Hewison M, Hellman P, Dralle H, Carling T, et al. 25-hydroxyvitaminD3-1alpha-hydroxylase expression in normal and pathological parathyroid glands. J Clin Endocrinol Metab 2002;87: 2967-72. 